\title{
Avaliação e desenvolvimento da compreensão em leitura no ensino fundamental
}

\author{
Acácia Aparecida Angeli dos Santos ${ }^{1}$ - Universidade São Francisco, Itatiba, Brasil \\ Evelin Zago de Oliveira - Universidade São Francisco, Itatiba, Brasil
}

\begin{abstract}
Resumo
O trabalho teve como objetivo investigar a adequação da técnica de Cloze para a avaliação e o desenvolvimento da compreensão em leitura. A primeira etapa foi realizada com 314 alunos de $2^{\mathrm{a}}$ a $4^{\mathrm{a}}$ séries do ensino fundamental e os resultados indicaram que o instrumento apresentava parâmetros psicométricos adequados. $\mathrm{Na}$ etapa seguinte, foram realizados dois programas de intervenção, o primeiro envolvendo 85 e o segundo 73 alunos de $3^{a}$ e $4^{a}$ s séries separados em grupos experimentais (GE) e de controle (GC), que responderam aos testes de Cloze validados, como pré e pós-testes. Os grupos experimentais participaram de sessões, nas quais foram apresentadas variações da técnica de Cloze, visando incrementar a compreensão da leitura. As análises indicaram superioridade do GE sobre o GC na situação de pós-teste apenas no segundo programa de intervenção. Sugerese que novos estudos sejam realizados com o intuito de testar a possibilidade de generalização dos resultados. Palavras-chave: Teste de Cloze, Compreensão de leitura, Ensino fundamental, Leitura.
\end{abstract}

\section{Assessment and development of reading comprehension with elementary school students}

\begin{abstract}
This work aimed at investigating the adequacy of Cloze technique to reading comprehension assessment and development. The first step was performed with 314 elementary school students attending from 2nd to 4th grades and the results indicated that the instrument showed appropriate psychometric parameters. In the following steps, two intervention programs were performed, the first one involving 85 and the second involving 73 rd and 4th grades students, separated in experimental group (EG) and control group (CG), who took validated Cloze tests, as pre- and post-tests. The experimental groups participated of sessions, in which variations of Cloze techniques were presented, in order to increase reading comprehension. Analyses indicated an advantage of EG upon CG on post-test situation only in the second intervention program. Accomplishment of new studies is suggested in order to test the possibility of generalization of the research results.

Keywords: Cloze Test, Reading comprehension, Elementary school, Reading.
\end{abstract}

Os avanços no campo da inteligência levaram ao desenvolvimento de modelos explicativos do desempenho captado por medidas objetivas (Carroll, 1978; Sternberg, 1987; entre outros), o que se repetiu, de forma similar, com a avaliação da compreensão em leitura (Palmer, MacLeod, Hunt \& Davidson, 1985; Daneman, 1991). Em razão da leitura se constituir de um complexo de habilidades cognitivas, algumas teorias passaram a ressaltar a preponderância de um ou outro componente do processo, enquanto outras enfatizaram o seu aspecto de multideterminação (Carr, 1981; Hannon \& Daneman, 2001; Mauad, Guedes \& Azzi, 2004).

Salientando o valor dos aspectos cognitivos, admite-se que uma nova informação adquirida pode ser usada produtivamente em outros contextos, o que exige que esse conhecimento esteja retido na memória. Estudos

\footnotetext{
${ }^{1}$ Endereço para Correspondência:

Programa de Pós-Graduação Stricto Sensu em Psicologia Universidade São Francisco. Rua Alexandre Rodrigues Barbosa, 45 - Centro - 13251-000 - Itatiba-SP

E-mail: acácia.santos@saofrancisco.edu.br
}

apontam que algumas pessoas são mais hábeis para reproduzir um texto, porém são inábeis para utilizar as informações contidas no texto para outros propósitos. Nesses casos, o conhecimento anterior ou prévio poderia explicar por que algumas pessoas compreendem mais rápida e eficazmente um texto do que outras (Kintsch 1988; Spires \& Donley, 1998). As evidências sobre a relevância dos papéis da aprendizagem e da memória na compreensão de leitura têm sido obtidas por meio de estudos experimentais que demonstram que leitores com alto nível de conhecimento anterior têm uma performance melhor, em um texto, do que leitores com um baixo nível de conhecimento anterior sobre o assunto focalizado (Kintsch, 1994; Van Dijk \& Kintsch, 1983).

Independentemente do quanto há de divergência entre os modelos teóricos de compreensão em leitura, há um alto grau de concordância a respeito da relevância do repertório de estratégias cognitivas e metacognitivas que o leitor possui para o processamento da informação contida no texto (Boruchovitch, 2001; Wade, Trathen \& Schraw, 1990). A utilização dessas estratégias permite que 
o leitor tenha a flexibilidade necessária para adaptar-se aos propósitos e às exigências da tarefa. Em virtude da importância atribuída à dimensão compreensão, faz-se indispensável discutir as possibilidades de como avaliá-la.

O interesse pela avaliação da compreensão da leitura cresceu com o fortalecimento do movimento para avaliação da inteligência e, em consequência, foi gerada uma grande quantidade de testes padronizados, muitos deles com boa acuidade. No entanto, em muitos casos, os testes de compreensão não estavam fundamentados teoricamente, o que dificultava inferir o que estavam medindo. Mesmo sem evidências de validade de construto, esses testes continuaram a ser utilizados por apresentarem algumas evidências de validade preditiva, além de bons índices de fidedignidade.

Decorrente do interesse mais geral pela avaliação da leitura, ampliou-se o interesse pelos testes diagnósticos, mesmo admitindo que seus resultados fornecem apenas uma amostra de comportamento relacionado à leitura. Considerando que ler é um comportamento complexo, composto por diversas habilidades e dificilmente avaliado na sua totalidade, entende-se que um bom instrumento de avaliação da compreensão em leitura deveria ter como função não só realizar uma estimativa do nível de compreensão dos alunos, mas também identificar suas dificuldades específicas.

O que se observa é que, embora existam vários testes para avaliar a habilidade de leitura, não é fácil mensurá-la, pois o seu produto final é a compreensão, que, por sua vez, não é um comportamento diretamente observável. Desse modo, ao afirmar que um estudante tem uma boa compreensão em leitura, o avaliador deve fundamentar-se em um teste que seja confiável e capaz de diferenciar o bom e o mau compreendedor. Um bom compreendedor deve ser hábil para abstrair as ideias do texto, contextualizar e possuir estratégias que lhe permitam recorrer aos elementos intra, inter e extratextuais para chegar à compreensão (Oliveira, Boruchovitch \& Santos, 2009).

Deve-se lembrar, ainda, que ao se pensar em avaliar um grande contingente de pessoas é necessário que se reflita sobre alguns aspectos práticos relacionados ao custo da elaboração, aplicação e correção. Muitos dos testes padronizados para os Estados Unidos, por exemplo, seriam extremamente onerosos para a realidade brasileira. No que tange a um instrumento confiável para o diagnóstico do nível de compreensão da leitura, a técnica de Cloze vem sendo muito utilizada, exatamente por ser de fácil manuseio e baixo custo (Santos, Boruchovitch \& Oliveira, 2009; Santos, Primi, Taxa \& Vendramini, 2002).

Criada por Taylor, em 1953, consiste, em sua formulação tradicional, na adaptação de um texto com aproximadamente duzentos vocábulos, do qual se omitem todos os quintos vocábulos, substituindo-os por um traço do tamanho da palavra omitida. Solicita-se ao leitor que, após a leitura integral do texto, complete os espaços em branco com a palavra que acredite ser a mais apropriada. Sua alta aceitação, para tal fim, já foi demonstrada pelas inúmeras publicações discorrendo sobre as diversas possibilidades de utilização desse recurso (Abraham \& Chapelle, 1992; Cunha, 2009; Kobayashi, 2002; Riley, 1986; Storey, 1997).

A técnica de Cloze está entre os primeiros procedimentos sistemáticos utilizados na avaliação da habilidade de leitura. É considerada adequada por favorecer a utilização dos dois principais tipos de processamento pelo leitor, que pode valer-se tanto da redundância semântica e sintática do texto, como de seus conhecimentos prévios. Ao lado disso, é um instrumento estruturado, simples e válido para se avaliar o nível de compreensão de leitura, podendo ser utilizado desde o ensino fundamental até o superior (Bensoussan, 1990; Santos, Boruchovitch \& Oliveira, 2009; Silva \& Witter, 2008).

Evidências de validade foram relatadas em estudos brasileiros como medida de inteligibilidade do texto (Molina, 1979), bem como um indicador da habilidade de leitura (Santos \& cols., 2002). Outro aspecto importante a ser observado é que, mesmo sendo um teste de construção e correção bastante simples, como já referido, sua utilização deve ser criteriosa, para que sejam evitados problemas graves em sua correção e, consequentemente, haja comprometimento no resultado obtido, invalidando-o como recurso para o uso diagnóstico.

O Cloze, enquanto teste, tem sido muito aceito como medida de avaliação da compreensão em leitura, sendo que Helfeldt e Henk (1985) estimavam que, nas décadas imediatamente anteriores ao estudo que realizaram, foram identificadas mais de 600 publicações discorrendo sobre as diversas possibilidades de utilização desse recurso. Storey (1997) destaca que a técnica de Cloze tem sido bastante discutida nos últimos 25 
anos por estudiosos da habilidade de compreensão em leitura. As discussões e pesquisas vão desde aquelas que investigam sua eficiência como medida de proficiência em uma língua estrangeira, até aqueles que o consideram como técnica para avaliação da habilidade de leitura na língua materna.

Para Bitar (1989), o Cloze possibilita ao leitor construir e reconstruir o texto continuamente, visto que, a cada acomodação da informação nova às informações já conhecidas, o leitor atribui uma nova contextualização e significado ao texto. Além disso, Kobayashi (2002) acrescenta que é uma técnica que pode ser utilizada como diagnóstico da compreensão em leitura, em textos de conteúdos diversos, de caráter geral ou específico de uma área do conhecimento.

Quanto à preparação do material para ser utilizado como um procedimento de Cloze, Abraham e Chapelle (1992) ponderam que a estruturação do texto deve ser de acordo com dois níveis de dificuldade relativos aos fatores intrínsecos e extrínsecos. Os fatores intrínsecos dependem de alguns elementos estruturais do texto, que facilitam a execução da tarefa e funcionam como um estímulo para o entendimento do item. Por sua vez, os fatores extrínsecos incluem os conhecimentos que o estudante tem sobre o assunto $\mathrm{e}$ as estratégias utilizadas para o desempenho da tarefa.

Corroborando as observações de Abraham e Chapelle (1992), a pesquisa de Santos e cols. (2002), valendo-se da análise da Teoria de Resposta ao Item, indicou que os acertos no Cloze não dependem somente da habilidade dos sujeitos, mas também das dificuldades dos itens que compõem o texto. Ressalta-se que com base no estudo dos itens do Cloze é possível abrir-se mais um leque de investigações científicas com resultados promissores e que permitirão a ampliação do conhecimento sobre esse instrumento.

Pesquisas estrangeiras têm apontado o teste de Cloze como preditivo do desempenho acadêmico (Alvarez, 1995; Barnitz, 1998; McKenna \& Kear, 1990; Oja, 1996). A ampla utilização da técnica de Cloze, tanto como procedimento de diagnóstico como de desenvolvimento da compreensão em leitura, denota o valor de sua utilização nos estudos que trazem resultados animadores.

No Brasil, muitos dos estudos utilizando o teste de Cloze têm sido realizados com universitários, alguns deles para avaliação da compreensão em leitura e, outros, em programas remediativos com estudantes que apresentam déficits de leitura (Sampaio \& Santos, 2002; Santos, 1997). Estudos com crianças e adolescentes também vêm sendo realizados, principalmente visando ao desenvolvimento de programas remediativos da compreensão (Santos, 2004). Os textos infantis, estruturados no padrão da técnica de Cloze, são elaborados com menor número de palavras e de omissões e têm sido utilizados como parâmetro da eficácia da intervenção realizada ou como procedimento específico para o desenvolvimento da compreensão com suas variações (Joly \& Istome, 2008; Oliveira \& Santos, 2008).

No entanto, a literatura brasileira recuperada mostrou escassez de estudos que abordassem o Cloze enquanto medida da compreensão e que explorassem a eficácia da técnica para o desenvolvimento da compreensão da leitura com crianças do ensino fundamental. Assim, o presente estudo foi proposto com o objetivo de estabelecer evidência de validade de critério, pela discriminação do desempenho dos participantes em razão da série cursada e para verificar o índice de precisão dos instrumentos por meio do método de teste e reteste e consistência interna. Também se propõe a analisar a eficácia da técnica para o desenvolvimento da compreensão da leitura pela comparação entre os resultados obtidos antes e depois de um programa de intervenção realizado em situação de sala de aula.

\section{Método}

\section{Etapa 1}

$\mathrm{Na}$ primeira etapa foi estabelecida a evidência de validade de critério e identificados os índices de precisão. Tal como sugerido por Taylor (1953), foram utilizados dois textos estruturados na forma do Cloze tradicional, com o $5^{\circ}$ vocábulo omitido. $\mathrm{Na}$ aplicação, os alunos foram orientados a preencher as lacunas com a palavra que julgassem mais adequada para completar o sentido do texto. A coleta de dados foi realizada em escolas da rede pública em cidades da região metropolitana de Campinas-SP em 314 crianças de $2^{\mathrm{a}}$ a $4^{\mathrm{a}}$ séries.

Os textos A princesa e o fantasma e Uma vingança infeliz foram especialmente elaborados por Santos (2005) para serem utilizados com crianças da faixa etária do ensino fundamental. A correção foi realizada com base na proposta de Bitar (1989), 
que considera como corretas somente as respostas que contêm palavras idênticas às omitidas. A pontuação máxima possível para cada teste é de 15 pontos, número correspondente à quantidade de lacunas contidas nos textos, perfazendo um total de 30 pontos possíveis.

Os resultados obtidos indicaram que os textos mostraram-se adequados para utilização na amostra pretendida. A análise da variância indicou haver diferença estatisticamente significativa entre as séries $[F(3,314)=55,75 ; p<0,001]$. Essa diferença foi justificada pelo teste de Tukey, que separou os escores das crianças nos grupos correspondentes às séries frequentadas. A análise da consistência interna apresentou índices de precisão satisfatórios, sendo o alfa de Cronbach de 0,83 para as crianças estudadas. Procedeu-se também a análise da consistência interna por série. Por essa análise obteve-se o índice para a segunda série de 0,85 , para a terceira série, de 0,69 e para a quarta série, de 0,72 .

\section{Etapa 2}

Constou de dois programas experimentais desenvolvidos nos segundos semestres de dois anos consecutivos. Os resultados de cada um desses programas, denominados Programa Experimental I e Programa Experimental II, serão apresentados separadamente conforme as descrições que se seguem.

\section{Programa Experimental I}

\section{Participantes}

Participaram do estudo 85 alunos de $4^{\mathrm{a}}$ série de uma escola pública da cidade de Jundiaí, no interior de São Paulo. Os participantes foram divididos em dois grupos, a saber, 42 (49\%) no grupo experimental e $50(51 \%)$ no grupo de controle. Do total, 51 eram do sexo masculino $(60 \%)$ e 34 do feminino $(40 \%)$. A idade variou de 9 a 12 anos, com média de 9,82 anos $(d p=0,46)$. É válido ressaltar que os alunos que faltaram a três sessões ou mais foram desconsiderados da amostra.

\section{Material}

Foram sete textos diferentes utilizados em cada uma das sessões consecutivas, além do texto utilizado nas sessões de pré e pós-teste "A princesa e o fantasma”, já mencionado anteriormente. Em cada sessão foi aplicado um texto diferente, estruturado segundo a técnica do Cloze gradual, na qual as dificuldades foram sendo progressivamente introduzidas. No item procedimento, as alterações dos textos de cada sessão serão mais bem detalhadas.

\section{Procedimento}

O estudo foi desenvolvido com a aprovação da direção da escola e a dos pais, por meio da assinatura do Termo de Consentimento Livre e Esclarecido e teve a duração de dois meses. Após a explicação dos objetivos do trabalho e a concordância de participação dos alunos, o programa ocorreu em nove sessões consecutivas, duas vezes por semana. A primeira sessão caracterizou-se pela aplicação do pré-teste em ambos os grupos, experimental e de controle, e a última também consistiu na aplicação do mesmo texto utilizado como pré-teste.

As sessões do programa de desenvolvimento ocorreram no período de seis semanas e foram aplicadas somente no grupo experimental, seguindo um padrão semelhante em todas elas. Assim, foram utilizados textos com diferentes padrões da técnica de Cloze (referidos no item material), sendo as instruções lidas em voz alta. O tempo foi o necessário para o preenchimento do texto e a correção imediata era feita pelos próprios alunos, com base na leitura íntegra feita pela aplicadora.

Os textos em Cloze diferenciaram-se em dificuldades gradualmente inseridas nas aplicações subsequentes. No primeiro texto, os alunos possuíam duas alternativas de respostas, apresentadas sob cada lacuna. No segundo, houve o oferecimento de três alternativas de respostas, também apresentadas embaixo de cada uma das lacunas. No terceiro texto utilizou-se a técnica de Cloze limitado, no qual todas as palavras retiradas do texto foram reagrupadas em ordem aleatórias na parte superior, acima do texto. O quinto texto incluiu um tracejado com o número exato de letras que cada uma das palavras omitida continha. No sexto, nenhuma pista adicional foi fornecida, havendo apenas a omissão dos décimos vocábulos, substituídos por um traço proporcional ao tamanho da palavra retirada.

Como último texto foi apresentada uma história estruturada com a omissão do sétimo vocábulo, não sendo dadas pistas adicionais para o preenchimento das lacunas. O programa de intervenção incluiu, ainda, a aplicação do pósteste, que consistia em responder o mesmo texto do pré-teste. Participaram do pré-teste ambos os grupos, experimental e controle. Vale ressaltar que nas sessões de pré e pós-teste os textos não foram 
corrigidos com os alunos e não lhes foi dado feedback imediato, diferentemente do que ocorreu nas sessões de intervenção.

Além de avaliações qualitativas dos tipos de erros cometidos (que será objeto de estudo em outro momento), os grupos foram comparados quantitativamente no pré e pós-teste, utilizando-se provas de estatística não-paramétrica. Assim, para a comparação intergrupos recorreu-se à prova não-paramétrica $U$ de Mann Whitney para comparar os desempenhos em ambas as situações. Os resultados constam da Tabela 1 .

Tabela 1 - Média dos ranks, e valores de z e p no pré-teste de ambos os grupos

\begin{tabular}{llcccc}
\hline Medidas & Grupos & $\mathrm{N}$ & $\begin{array}{c}\text { Média } \\
\text { dos ranks }\end{array}$ & $\mathrm{z}$ & $\mathrm{p}$ \\
\hline Pré-Teste & Grupo experimental & 42 & 45,63 & $-0,987$ & 0,324 \\
& Grupo controle & 43 & 40,43 & & \\
Pós-Teste & Grupo experimental & 42 & 50,94 & $-2,949$ & 0,003 \\
& Grupo controle & 43 & 35,24 & & \\
\hline
\end{tabular}

Os resultados não revelaram diferença significativa entre os grupos na medida do préteste. No entanto, no pós-teste verificaram-se diferença altamente significativas entre os dois grupos. Importante lembrar que apenas os integrantes do grupo experimental foram submetidos ao programa de intervenção.

O teste de Sinal foi o escolhido para a comparação do efeito do programa de intervenção. Os resultados obtidos estão apresentados na Tabela 2.

Tabela 2 - Comparação de amostras dependentes pelo teste de Sinal

\begin{tabular}{|c|c|c|c|c|c|}
\hline Grupos & & & $\mathrm{N}$ & $z$ & $\mathrm{p}$ \\
\hline \multirow[t]{4}{*}{ Grupo experimental } & Pré-teste & Diferenças negativas a & 27 & & \\
\hline & vs & Diferenças positivas ${ }^{b}$ & 8 & $-3,043$ & 0,002 \\
\hline & Pós-teste & Empates ${ }^{c}$ & 7 & & \\
\hline & & $\mathrm{n}$ & 42 & & \\
\hline \multirow[t]{4}{*}{ Grupo controle } & Pré-teste & Diferenças negativas a & 35 & & \\
\hline & vs & Diferenças positivas ${ }^{b}$ & 4 & $-4,804$ & 0,000 \\
\hline & Pós-teste & Empates ${ }^{c}$ & 4 & & \\
\hline & & $\mathrm{n}$ & 43 & & \\
\hline
\end{tabular}

a: Pós-teste<Pré-teste; b: Pós-teste $>$ Pré-teste; c: Pós-teste=Pré-teste

Os dados relativos à comparação intragrupos demonstraram uma queda de desempenho tanto no grupo experimental $(\mathrm{n}=27)$ quanto no grupo controle $(\mathrm{n}=35)$. Também para um número equivalente de alunos não houve alteração no desempenho (grupo experimental $n=7$ e grupo controle $n=4$ ) nas medidas realizadas no pré e no pós-teste.

\section{Discussão dos resultados do Programa Experimental I}

Pelos dados obtidos no pré-teste verificouse que os grupos, experimental e de controle, possuíam uma média de classificação inicial muito próxima, pela qual se constatou que não havia diferença significativa entre os grupos. Assim, identificou-se que eles possuíam nível similar de compreensão em leitura.

$\mathrm{Na}$ comparação intragrupo não foi verificada diferença significativa entre os grupos entre o pré e o pós-teste no grupo experimental, diferentemente do esperado. No entanto, vale ressaltar que na comparação intergupos apareceu diferença significativa entre o grupo experimental e o de controle, relacionada ao pós-teste, mostrando um desempenho superior do grupo experimental, que não existia na ocasião do préteste. Assim sendo, pode-se afirmar que houve diferenças quantitativas entre os grupos, como já apontado por pesquisadores da área, tanto estrangeiros como nacionais (Bensoussam, 1990; Bernitz, 1998; Bitar, 1989; Oja, 1996; Santos, 2004; Storey, 1997).

Dessa forma, os resultados obtidos neste estudo estão condizentes com os demais 
apresentados na literatura, que consideram o teste de Cloze um procedimento diagnóstico e de desenvolvimento da compreensão em leitura $\mathrm{O}$ grande número de pesquisas que utilizam a técnica de Cloze somente atesta a qualidade do instrumento, não apenas para o diagnóstico da compreensão de leitura, mas como uma ferramenta de intervenção e desenvolvimento psicoeducacional. Nesse sentido acredita-se ser importante desenvolver pesquisas voltadas ao ensino fundamental (Alvarez, 1995; Chance, 1985; Giordano, 1985; Joly \& Istome, 2008; McKenna \& Kear, 1990; Riley, 1986).

No entanto, a comparação intragrupo não evidenciou a diferença esperada em termos quantitativos. Por motivos não identificados, que podem ter sido apenas circunstanciais, tanto os alunos do grupo experimental como os alunos no grupo de controle apresentaram um desempenho mais baixo no pós-teste. Importante ressaltar que com base em observação assistemática e nos depoimentos dos professores, constatou-se que qualitativamente o grupo experimental era superior ao de controle, mostrando mais interesse pelas atividades de leitura, o que parece ter sido resultado da participação no programa. Entendeuse, ainda, como benefício decorrente deste estudo, que foi demonstrada aos professores a possibilidade de se utilizar em sala de aula uma técnica de fácil manejo que se configura como alternativa para o desenvolvimento de compreensão da leitura. Pode-se, assim, afirmar que é viável o uso do Cloze como ferramenta que auxilia os alunos a desenvolver a habilidade de leitura, considerada essencial para o aprendizado dos demais conteúdos ensinados nessa etapa de escolarização.

Examinando os resultados alcançados, julgou-se necessária a ampliação do programa de intervenção. Considerou-se que um maior número de sessões de treino poderia acentuar a tendência dos dados obtidos pelos alunos que participaram do programa, que se apresentaram qualitativamente superiores na compreensão em leitura. Ao lado disso, também se aquilatou que seria interessante incluir alunos de série anterior à $4^{\mathrm{a}}$, visto que desde a primeira aplicação do préteste a média obtida pelos alunos foi considerada alta (acima do valor da mediana dos pontos que poderiam ser alcançados), lembrando que o máximo eram 15 pontos. No ano seguinte o programa foi repetido, mantendo-se as suas características básicas, mas incluindo também alunos de $3^{\text {a }}$ série e ampliando-se o número de sessões do programa de intervenção, tal como relatado a seguir.

\section{Programa Experimental II}

\section{Participantes}

Foram 73 alunos de $3^{a}(n=36)$ e $4^{a}(n=37)$ séries de uma escola pública da cidade de JundiaíSP, divididos em dois grupos de acordo com a série. O grupo experimental da $3^{\mathrm{a}}$ série era formado por 17 alunos $(51 \%)$ e o da $4^{\mathrm{a}}$ série por 16 alunos $(49 \%)$. O grupo de controle foi composto por 19 alunos da $3^{\text {a }}$ série $(48 \%)$, e 21 alunos compuseram o grupo de controle da $4^{\mathrm{a}}$ série $(52 \%)$. Dentre todos os participantes, 31 eram do sexo masculino $(42 \%)$ e 42 do feminino $(57 \%)$. A média de idade foi de 9,3 anos, variando de 8 a 10, e desvio padrão de 0,68 .

\section{Material}

Em cada sessão foram aplicados textos diferentes, estruturados segundo a Técnica de Cloze Gradual, nos quais houve a inclusão de dificuldades inseridas progressivamente. Neste segundo programa houve uma repetição de cada uma das variações, dado o número maior de sessões propostas. Pretende-se que na leitura do procedimento as variações utilizadas nas aplicações deixem as diferenças entre as sessões bem evidentes.

\section{Procedimento}

$\mathrm{Na}$ segunda etapa houve os mesmos cuidados éticos tomados na primeira. Assim, somente após a aprovação da escola e o TCLE assinado pelos pais o estudo foi desenvolvido. Também a primeira sessão, que se caracterizava pela aplicação de dois textos em Cloze (pré-teste) em ambos os grupos, experimental e de controle, foi realizada após a explicação e a adesão dos alunos presentes.

As sessões foram realizadas ao longo de cinco meses, uma vez por semana, e foram aplicadas somente no grupo experimental, seguindo-se um padrão semelhante em todas as sessões. $\mathrm{Na} 2^{\mathrm{a}}$ e $3^{\mathrm{a}}$ sessões do GE foram aplicados dois textos, com histórias diferenciadas, com omissão do $10^{\circ}$ vocábulo, nos quais duas alternativas que completavam a lacuna foram apresentadas, sendo apenas uma a correta. A resposta errada estava bem evidente, apresentando discrepância sintática e semântica em relação à prevista para o espaço. Já nas $4^{a}$ e $5^{a}$ sessões os dois textos também continham omissões no $10^{\circ}$ 
vocábulo, mas havia três alternativas para o preenchimento da lacuna, sendo apenas uma a correta. Dentre as respostas erradas incluem-se: (a) uma primeira alternativa com erro bem evidente, com discrepância sintática e semântica em relação à prevista e (b) a segunda, com discrepância sintática ou semântica em relação à esperada para o preenchimento adequado do espaço.

Nas $6^{a}$ e $7^{a}$ sessões foram utilizadas histórias ainda com omissão do $10^{\circ}$ vocábulo, mas com a lista das palavras omitidas no texto entregue anexa a ele, tendo sido listadas aleatoriamente e repetidas tantas vezes, quantas fosse a sua presença no texto. Outros dois textos com conteúdos diferentes e omissão do $10^{\circ}$ vocábulo foram empregados na $8^{a}$ e $9^{a}$ sessões, cada traço das lacunas trazendo grafada a letra inicial da palavra omitida. Continuou-se ainda a omitir o $10^{\circ}$ vocábulo nos textos específicos das sessões $10^{\mathrm{a}} \mathrm{e}$ $11^{a}$, nas quais as lacunas foram substituídas por uma linha de pequenos traços correspondendo a cada letra da palavra omitida.

Por fim, na $12^{\mathrm{a}}$ e $13^{\mathrm{a}}$ sessões um texto diferente em cada uma delas foi estruturado segundo a Técnica de Cloze, respectivamente, com omissão do $10^{\circ}$ e do $7^{\circ}$ vocábulo, não sendo fornecida nenhuma pista adicional para o preenchimento dos espaços em branco. $\mathrm{Na} 14^{\mathrm{a}}$ sessão, utilizada como pós-teste, as mesmas histórias do pré-teste foram reaplicadas.

Vale destacar que para cada sessão do programa de intervenção foram fornecidas instruções específicas, dependendo do tipo de atividade solicitada. Após a finalização da tarefa os alunos recebiam uma nova cópia do texto, ainda com as lacunas, para que a correção fosse feita por eles mesmos, mediante a leitura do texto pela aplicadora. Na última sessão, a do pós-teste, não foi dado feedback das respostas corretas.

\section{Resultados do Programa Experimental II}

Os dados coletados foram analisados em função dos objetivos pretendidos, portanto, foram submetidos às provas estatísticas descritivas e provas não-paramétricas, a saber, teste de Mannwhitney para a comparação entre grupos e teste de Wilcoxon Signed Ranks, para a comparação intragrupos.

A fim de explorar melhor a diferença entre os grupos, foi realizada a prova de análise de variância não-paramétrica de Mann-Whitney. Os resultados estão apresentados na Tabela 3 .

Tabela 3 - Pontuações médias por ranks, valor de $U$ e $p$ dos alunos no pré-teste, por série e grupo

\begin{tabular}{|c|c|c|c|c|c|c|}
\hline Série & Pré -Teste & Grupos & $\mathrm{N}$ & M por & $\mathrm{U}$ & $\mathrm{p}$ \\
\hline \multirow{4}{*}{$3^{\mathrm{a}}$ série } & \multirow{2}{*}{ A princesa e o fantasma } & Experimental & 17 & 16,53 & \multirow{2}{*}{128,0} & \multirow{2}{*}{0,300} \\
\hline & & Controle & 19 & 20,26 & & \\
\hline & \multirow{2}{*}{ Uma vingança infeliz } & Experimental & 17 & 17,74 & \multirow{2}{*}{148,5} & \multirow{2}{*}{0,684} \\
\hline & & Controle & 19 & 19,18 & & \\
\hline \multirow{4}{*}{$4^{\mathrm{a}}$ série } & \multirow{2}{*}{ A princesa e o fantasma } & Experimental & 16 & 14,97 & \multirow{2}{*}{103,5} & \multirow{2}{*}{0,047} \\
\hline & & Controle & 21 & 22,07 & & \\
\hline & \multirow{2}{*}{ Uma vingança infeliz } & Experimental & 16 & 18,47 & \multirow{2}{*}{159,5} & \multirow{2}{*}{0,797} \\
\hline & & Controle & 21 & 19,40 & & \\
\hline
\end{tabular}

No que se refere ao pré-teste, pode-se observar que tanto na terceira como na quarta série, ambos os grupos tiveram desempenho similar, mas sempre com alguma superioridade para o grupo de controle. Observa-se, contudo, que na $4^{a}$ série o resultado do grupo de controle no texto "A princesa e o fantasma" foi significativamente superior.
Visando analisar as diferenças intragrupos, foi utilizada a prova de Wilcoxon Signed Ranks (Teste de Sinal $Z$ ), o qual apresenta o número de sujeitos que melhoraram seu desempenho, quantos permaneceram na mesma posição e quantos pioraram em desempenho após o término do programa de intervenção. Os resultados do grupo experimental estão apresentados na Tabela 4. 
Tabela 4 - Comparação de amostras dependentes teste de Sinal $Z$ para o GE

\begin{tabular}{|c|c|c|c|c|c|c|c|}
\hline Série & Grupo Exper & Imental & Ranks & $\mathrm{N}$ & $\begin{array}{l}\text { M por } \\
\text { Ranks }\end{array}$ & Z & $\mathrm{p}$ \\
\hline \multirow{6}{*}{$3^{a}$ série } & \multirow{3}{*}{$\begin{array}{l}\text { A princesa e o fantasma } \\
\text { A princesa e o fantasma }\end{array}$} & \multirow{3}{*}{$\begin{array}{l}\text { (Pós - Teste) } \\
\text { (Pré - Teste) }\end{array}$} & Negativos & 3 & 5,83 & & \\
\hline & & & Positivos & 12 & 8,54 & $-2,819$ & 0,005 \\
\hline & & & Empates & 0 & & & \\
\hline & \multirow{3}{*}{$\begin{array}{l}\text { Uma vingança infeliz } \\
\text { Uma vingança infeliz }\end{array}$} & \multirow{3}{*}{$\begin{array}{l}\text { (Pós - Teste) } \\
\text { (Pré -Teste) }\end{array}$} & Negativos & 3 & 6,17 & & \\
\hline & & & Positivos & 10 & 7,25 & $-1,994$ & 0,046 \\
\hline & & & Empates & 2 & & & \\
\hline \multirow{6}{*}{$4^{a}$ série } & \multirow{3}{*}{$\begin{array}{l}\text { A pri ncesa e o fantasma } \\
\text { A Princesa e o Fantasma }\end{array}$} & \multirow{3}{*}{$\begin{array}{l}\text { (Pós -Teste) } \\
\text { (Pré - Teste) }\end{array}$} & Negativos & 2 & 5,75 & & \\
\hline & & & Positivos & 9 & 6,06 & $-1,835$ & 0,067 \\
\hline & & & Empates & 2 & & & \\
\hline & \multirow{3}{*}{$\begin{array}{l}\text { Uma vingança infeliz } \\
\text { Uma vingança infeliz }\end{array}$} & \multirow{3}{*}{$\begin{array}{l}\text { (Pós - Teste) } \\
\text { (Pré -Teste) }\end{array}$} & Negativos & 2 & 2,00 & & \\
\hline & & & Positivos & 10 & 7,40 & $-3,066$ & 0,002 \\
\hline & & & Empates & 1 & & & \\
\hline
\end{tabular}

$\mathrm{Na}$ comparação do grupo experimental por série, observa-se que os alunos da terceira série, em ambos os pré e pós-testes, apresentaram melhora significativa de desempenho. Já os alunos da quarta série apresentaram melhoras significativas somente no pré e pós-teste do texto "Uma vingança infeliz", visto que no texto "A princesa e o fantasma" a diferença foi marginalmente significativa.

$\mathrm{Na}$ Tabela 5 estão apresentados os dados referentes ao grupo controle. Utilizou-se o teste de sinal $Z$ para a comparação de amostras dependentes de cada um dos grupos de $3^{\mathrm{a}} \mathrm{s}$ e $4^{\mathrm{a}} \mathrm{s}$ séries.

Tabela 5 - Comparação de amostras dependentes - teste de Sinal Z para o GC

\begin{tabular}{|c|c|c|c|c|}
\hline Série & Grupo Controle & Ranks $\mathrm{N}$ & $\mathrm{J}$ M por & Z \\
\hline \multirow{6}{*}{$\begin{array}{l}3^{\mathrm{a}} \\
\text { série }\end{array}$} & \multirow{3}{*}{$\begin{array}{l}\text { A princesa e o fantasma (Pós-Teste) } \\
\text { A princesa e o fantasma (Pré-Teste) }\end{array}$} & Negativos 8 & $8 \quad 5,50$ & \multirow{3}{*}{$-0,9230,356$} \\
\hline & & Positivos & 10,86 & \\
\hline & & Empates & 4 & \\
\hline & \multirow{3}{*}{$\begin{array}{l}\text { Uma vingança infeliz (Pós-Teste) } \\
\text { Uma vingança infeliz (Pré-Teste) }\end{array}$} & Negativos & 4,88 & \multirow{3}{*}{$-2,5320,011$} \\
\hline & & Positivos 12 & 9,71 & \\
\hline & & Empates & 3 & \\
\hline \multirow{6}{*}{$\begin{array}{l}4^{\mathrm{a}} \\
\text { série }\end{array}$} & \multirow{3}{*}{$\begin{array}{l}\text { A princesa e o fantasma (Pós-Teste) } \\
\text { A princesa e o fantasma (Pré-Teste) }\end{array}$} & Negativos & 7,38 & \multirow{3}{*}{$-0,8490,396$} \\
\hline & & Positivos & 10,44 & \\
\hline & & Empates & 4 & \\
\hline & \multirow{3}{*}{$\begin{array}{l}\text { Uma vingança infeliz (Pós-Teste) } \\
\text { Uma vingança Infeliz (Pré-Teste) }\end{array}$} & Negativos 2 & 16,25 & \multirow{3}{*}{$-2,5360,011$} \\
\hline & & Positivos 17 & 9,26 & \\
\hline & & Empates & 2 & \\
\hline
\end{tabular}

As análises realizadas permitiram inferir que nos grupos de controle, tanto da terceira quanto da quarta série, os alunos apresentaram melhoras significativas relativas ao texto "Uma vingança infeliz", mesmo não participando do programa de intervenção. Esses resultados serão mais bem explorados na discussão que se segue.

\section{Discussão dos resultados do Programa Experimental II}

Os resultados obtidos revelaram que o grupo experimental apresentou pontuações altas em todas as sessões, independente de série, o que mais uma vez revela a eficácia da técnica de Cloze 
como instrumento diagnóstico de compreensão em leitura, corroborando assim os resultados obtidos por pesquisadores nacionais e internacionais (Bensoussam, 1990; Bitar, 1989; Storey, 1997; Santos, 2004).

Ao comparar os grupos em relação ao préteste, pode-se observar que as pontuações médias do grupo controle eram superiores ao grupo experimental. $\mathrm{Na}$ terceira série as médias foram superiores em ambos os textos, porém não apresentam diferenças significativas. Já na quarta série, somente o texto "A princesa e o fantasma", apresentou médias significativamente maiores. Essa diferença pode ser explicada pelo fato de o grupo experimental ser formado pelas turmas que apresentavam mais dificuldades de leitura, segundo a direção da escola. No que se refere ao pós-teste, ambas as séries apresentaram aumento em suas médias, porém as diferenças não apresentavam significância estatística.

Em um segundo momento, a comparação intragrupos, de ambas as séries, revelou uma melhora significativa do grupo experimental, pois as médias por ranks apresentaram que um grande número de alunos obteve melhoras do pré para o pós-teste. Somente na quarta série, no pré e pósteste do texto "A princesa e o fantasma" não foram observadas tais diferenças. No que se refere ao grupo controle, a comparação revelou que mesmo não participando do programa de intervenção, os alunos, em sua maioria, apresentaram melhora em sua compreensão em leitura. As diferenças entre as médias foram significativas para a terceira e quarta séries, no pré e pós-teste do texto "Uma vingança infeliz". Este fato pode ser explicado pelo bom desempenho apresentado pelos grupos controle, já no pré-teste, mas cria possibilidades para futuros estudos que possam explorar este aspecto, ou seja, a fato dos alunos já possuírem uma boa compreensão de leitura.

\section{Considerações finais}

O estudo conduzido foi importante não só pelos resultados imediatamente alcançados com o programa de intervenção, mas também por outras consequências educacionais mais difíceis de serem mensuradas. Assim, este trabalho de pesquisa teve também o efeito de gerar na equipe da escola (professores e coordenação, principalmente) um interesse pelos resultados alcançados e a abertura de perspectivas para incluir na rotina escolar outras formas de trabalho que incentivem a utilização de caminhos alternativos para estimular os alunos a lerem com mais frequência e de maneira compreensiva. No entanto, o empreendimento de levar a cabo pesquisas de intervenção é árduo e demanda dos pesquisadores uma constante revisão do projeto e muita atenção às variáveis intervenientes que interferem na proposta inicialmente concebida.

Mediante os dados apresentados é válido ressaltar a importância de técnica de Cloze como instrumento diagnóstico e remediativo de compreensão em leitura, e sua relevância no contexto educacional. Contudo, sabe-se que há ainda lacunas no conhecimento referentes às possíveis relações entre a compreensão da leitura e outras habilidades básicas, o que necessita ser mais bem explorado em novos estudos.

Sugere-se que estudos futuros investiguem tais relações, especialmente no que diz respeito a algumas habilidades específicas, como a consciência fonológica, a consciência morfológica, o reconhecimento de palavras e a escrita de palavras ditadas, entre outras. É reconhecida a importância que essas habilidades têm no desempenho escolar. Constata-se que no Brasil não há instrumentos de avaliação com evidências de validade para serem usados de forma a identificar eventuais desajustes, bem como possibilitar o diagnóstico preventivo de possíveis dificuldades de aprendizagem.

\section{Referências}

Abraham, R. G. \& Chapelle, C. A. (1992). The meaning of cloze test scores: an item difficulty. The Modern Language Journal, 76, 468479 .

Alvarez, O. H. (1995). Propuesta alternativa para estimular la comprensión lectora: una experiencia con niños de $5^{\circ}$ grado. Lectura $y$ Vida, 19(1), 51-55.

Barnitz, J. G. (1998). Linguistic perspectives in literacy education. The Reading Teacher, 580(2), 110-117.

Bensoussan, M. (1990). Redundancy and the cohesion Cloze. Journal of Research in Reading, 13(1), 18-37.

Bitar, M. L. (1989). Eficiência dos instrumentos de avaliação em leitura. Dissertação de Mestrado, PUC-São Paulo, São Paulo.

Boruchovitch, E. (2001). Algumas estratégias de compreensão em leitura de alunos do ensino 
fundamental. Psicologia Escolar e Educacional, 5 (1), 19-25.

Carr, T. H. (1981). Building theories of reading ability: on the relation between individual differences in cognitive skills and reading comprehension. Cognition, 9, 73-114.

Carroll, J. B. (1978). How shall we study individual differences in cognitive abilities? Methodological and theoretical perspectives. Intelligence, 2, 73-115.

Chance, L. (1985). Use Cloze encounters of the readability kind for secondary school students. Journal of Reading, 29(8), 690-693.

Cunha, N. B. (2009). Pesquisas com o teste de cloze no Brasil. Em A. A. A. Santos, E. Boruchovitch \& K. L. Oliveira (Orgs.). Cloze: um instrumento de diagnóstico e intervenção (pp. 79118). São Paulo: Casa do Psicólogo.

Daneman, M. (1991). Individual differences in reading skill. Em R. Barr, M. L. Kamil, P. Mosenthal \& P. D. Pearson (Orgs.). Handbook of reading research (Vol. 2, pp. 512-538). Nova Iorque: Longman.

Giordano, G. (1985). Learning to read erased text. Academic Therapy, 20(3), 317-322.

Hannon, B. \& Danemon, M. (2001). A new tool for measuring and understanding individual differences in the component processes of reading comprehension. Journal of Educational Psychology, 93(1), 103-128.

Helfeldt, J. P. \& Henk, W. A. (1985). Unfulness of conventional vs total random cued cloze tests as measure of reading comprehension. Journal of Reading, 28(8), 719-725.

Joly, M. C. R. A. \& Istome, A. C. (2008). Compreensão em leitura e capacidade cognitiva: Estudo de validade do teste de Cloze Básico - MAR. Psic, 9(2), 219-228.

Kintsch, W. (1988). The role of knowledge in discourse comprehension a construction integration model. Psychological Review, 95(2), 163-182.

Kintsch, W. (1994). Text comprehension, memory and learning. American Psychologist, 49(4), 294303.

Kobayashi, M. (2002). Cloze tests revisited: exploring item characteristics with special attention to scoring methods. Modern Language Journal, 86(4) 571-586.

Mauad, L. C., Guedes, M. C. \& Azzi, R. G. (2004). Análise do comportamento e a habilidade de leitura: um levantamento crítico de artigos do JABA. Psico-USF, 9(1), 59-69.

McKenna, M. C. \& Kear, D. J. (1990). Measuring attitude toward reading: a new tool for teachers. The Reading Teacher, 23, 626-639.

Molina, O. (1979). Avaliação da inteligibilidade de livros didáticos de $1^{\circ}$ e $2^{\circ}$ graus por meio da técnica de Cloze. Tese de Doutorado, Universidade de São Paulo, São Paulo.

Oja, L. A. (1996). Using story to develop reading comprehension. Journal of Adolescent \& Adult Literacy, 40(2), 129-130.

Oliveira, K. L. \& Santos, A. A. A. (2008). Estudo de intervenção para a compreensão em leitura e produção de textos na universidade. Interação (Curitiba), 12(2)169-177.

Oliveira, K. L., Boruchovitch, E. \& Santos, A. A. A. (2009). A técnica de cloze na avaliação da compreensão em leitura. Em A. A. A. Santos, E. Boruchovitch \& K. L. Oliveira (Orgs.). Cloze: um instrumento de diagnóstico e intervenção (pp. 47-78). São Paulo: Casa do Psicólogo.

Palmer, J., MacLeod, C. M., Hunt, E. \& Davidson, J. E. (1985). Information processing correlates of reading. Journal of Memory and Language, 24, 59-88.

Riley, J. D. (1986). Progressive Cloze as a remedial technique. The Reading Teacher, 39(6), 576-581.

Sampaio, I. S. \& Santos, A. A. A. (2002). Leitura e redação entre universitários: avaliação de um programa de remediação. Psicologia em Estudo, 7 (1), 31-38.

Santos, A. A. A. (1997). Psicopedagia no $3^{\circ}$ grau: avaliação de um programa de remediação em leitura e estudo. Pro-Posições, 8(1), 27-37.

Santos, A. A. A. (2004). O Cloze como técnica de diagnóstico e remediação da compreensão em leitura. Interação em Psicologia, 8(2) 217-226.

Santos, A. A. A., Boruchovitch, E. \& Oliveira, K. L. (2009). Cloze: um instrumento de diagnóstico e intervenção. São Paulo: Casa do Psicólogo.

Santos, A. A. A., Primi, R., Taxa, F. \& Vendramini, C. M. M. (2002). O teste de Cloze na avaliação 
da compreensão em leitura. Psicologia: Reflexão e Critica, 15(3), 549-560.

Silva, E, M. T. \& Witter, G. P. (2008). Compreensão de texto e desempenho acadêmico em estudantes de psicologia. Estudos de Psicologia, Campinas, 25(3), 305-403.

Spires, H. A. \& Donley, J. (1998). Prior knowledge activation: inducing engagement with informational texts. Journal of Educational Psychology, 90(2), 249-260.

Sternberg, R. J. (1987). The psychology of verbal comprehension. Em R. Glaser (Org.). Advances in instructional psychology (pp. 97-151). Hillsdale, NJ: Erlbaum.

Storey, P. (1997). Examining the test-talking process: a cognitive perspective on the discourse cloze test. Language testing, 14(2), 214-231.

Taylor, W. L. (1953). Cloze procedure: a new tool for measuring read-ability. Journalism Quarterly, 30, 415-433.

Van Dijk, T. A. \& Kintsch, W. (1983). Strategies in discourse comprehension. Nova Iorque: Academic Press.

Wade, S. E., Trathen, W. \& Schraw, G. (1990). An analysis of spontaneous study strategies. Reading Research Quarterly, 25, 147-166.

Recebido em junho de 2009 Reformulado em outubro de 2009 Aprovado em dezembro de 2009

Sobre as autoras:

Acácia Aparecida Angeli dos Santos é doutora em Psicologia Escolar e do Desenvolvimento Humano pela USP, professora titular da graduação e Pós-Graduação Stricto Sensu em Psicologia da USF e bolsista produtividade do CNPq.

Evelin Zago de Oliveira é psicóloga graduada pela Universidade São Francisco, Itatiba-SP. 